\title{
PALINOLOGÍA DE LA FORMACIÓN MONTE CHICO (CRETÁCICO TARDÍO) DE LA PROVINCIA DE SANTA CRUZ, ARGENTINA: GIMNOSPERMAS
}

\author{
LETICIA POVILAUSKAS \\ División Paleobotánica, Museo Argentino de Ciencias Naturales "Bernardino Rivadavia", Av. Ángel Gallardo 470, \\ C1405DJR, Buenos Aires, Argentina.lepovilauskas@macn.gov.ar
}

\begin{abstract}
PALYNOLOGY OF THE MONTE CHICO FORMATION (LATE CRETACEOUS) FROM SANTA CRUZ PROVINCE, ARGENTINA: POLLEN OF GYMNOSPERMS. Palynological assemblages composed by continental and marine palynomorphs were recovered from the Monte Chico Formation, late Cretaceous, Santa Cruz Province. In this contribution palynological assemblages of gymnosperms were studied. The samples were taken from the all section of this unit. Sixteen species of pollen grains were here determined. They belong to the genera: Araucariacites, Classopollis, Cycadopites, Dacrycarpites, Gamerroites, Lygistepollenites, Microcachyridites, Phyllocladidites, Podocarpidites and Trisaccites. On the basis of the stratigraphic distribution of the identified species, mainly in Argentina and Antarctica, and joined with the preliminary analysis determinate of spores, a Maastrichtian-Late Maastrichtian age is here suggested. The palynological assemblages indicate a typically of shore environment of deposition, with continental conditions towards to the most upper part of section.
\end{abstract}

Key words: gymnosperms, Monte Chico Formation, Cretaceous, Argentina.

RESUMO - As assembleias palinológicas recuperadas dos sedimentos da Formação Monte Chico, Cretáceo superior, Província de Santa Cruz, apresentaram elementos marinhos (cistos de dinoflagelados) e continentais. Nessa contribuição foram estudadas as associações constituídas por grãos de pólen de gimnospermas. As amostras foram recuperadas ao longo de toda a secção. Foram descritas dezesseis espécies dos gêneros: Araucariacites, Classopollis, Cycadopites, Dacrycarpites, Gamerroites, Lygistepollenites, Microcachyridites, Phyllocladidites, Podocarpidites e Trisaccites. Com base na distribuição estratigráfica das espécies identificadas, principalmente na Argentina e na Antártida, e juntamente com a análise dos esporos realizada anteriormente, sugere-se a idade Maastrichtiano a Maastrichtiano superior. A aglutinação palinológica indica um ambiente deposicional tipicamente litorâneo, com continentalização progressiva para a parte superior da secção.

Palavras-chave: gimnospermas, Formação Monte Chico, Cretáceo, Argentina.

\section{INTRODUCCIÓN}

En esta contribución se continúa con el estudio sistemático de los palinomorfos de la Formación Monte Chico, Maastrichtiano de la Provincia de Santa Cruz, Argentina, obtenidos de secciones aflorantes en dos zonas de estudio, próximo al límite con Chile (Figura 1). El área de estudio se encuentra ubicada en el extremo sudoccidental de la Provincia de Santa Cruz, en las inmediaciones de la ciudad de Río Turbio. El muestreo palinológico fue realizado en varias secciones ubicadas en los afloramientos próximos a la zona de Cancha Carrera ( $51^{\circ} 11^{\prime} 20,2^{\prime}$ 'S, $\left.72^{\circ} 20^{\prime} 55,5^{\prime \prime} \mathrm{O}\right)$, y en el área del cerro de la cruz $\left(51^{\circ} 33^{\prime} 00,5^{\prime}\right.$ 'S, $72^{\circ} 25^{\prime} 43,2^{\prime \prime}$ 'O), conformando un perfil integrado de toda la secuencia analizada (Figura 2).

La Formación Monte Chico está integrada por areniscas finas a medianas, castaño claras a castaño rojizas y gris verdosas, en parte calcáreas, con abundantes concreciones gris oscuras que incluyen una megafauna de invertebrados fósiles; fangolitas, limonitas con intercalaciones coquinoides y conglomerados portadores de megafósiles (Malumián \& Panza, 1996) (Figura 3).
Trabajos preliminares sobre secuencias cretácicas cerca del área de estudio permitieron sugerir un ambiente de depositación marino litoral cercano a la línea de costa, y una edad en el entorno del Maastrichtiano-Maastrichtiano tardío para la Formación Monte Chico.

En el presente análisis se describe sistemáticamente los granos de polen pertenecientes a las gimnospermas de dicha unidad, junto a las algas de agua dulce, que permiten realizar inferencias paleoambientales para esta formación.

\section{MATERIAL Y MÉTODOS}

Para la toma de las muestras palinológicas se eligieron las zonas con las mejores exposiciones llegando a un total de 36 niveles pelíticos y arenopelíticos muestreados en las dos zonas de estudio (Estancia San José y Estancia Laguna Salada) (Figura 2). El distanciamiento entre muestras fue irregular, dependiendo de la litología y de los tramos cubiertos. En intervalos convenientes el muestreo se efectuó a un distanciamiento de 5 metros. El material recolectado fue embolsado y convenientemente rotulados para su posterior análisis en el laboratorio. 

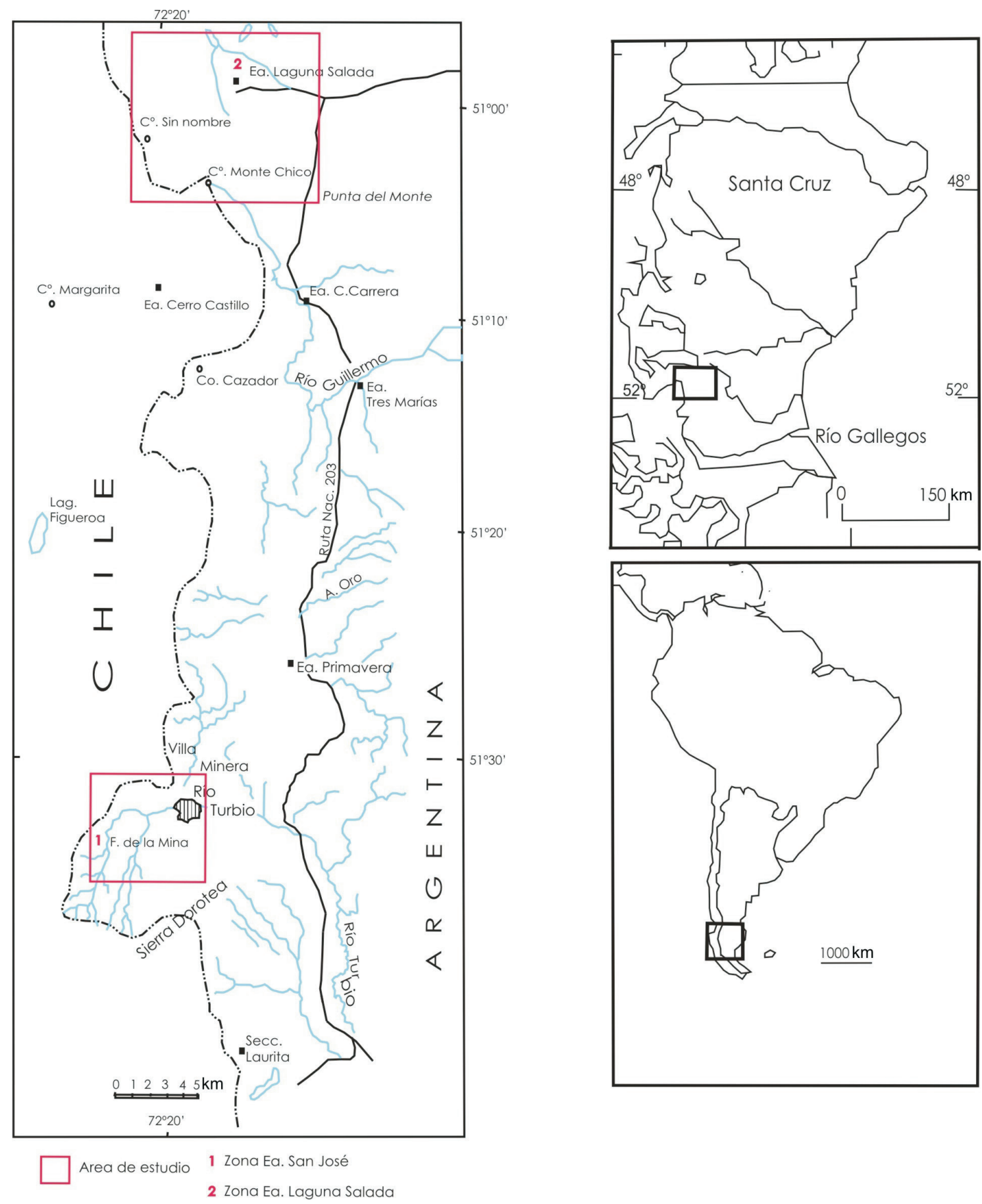

Figura 1. Mapa de ubicación geográfica.

Figure 1. Geographic location map.

La extracción palinológica se realizó siguiendo métodos convencionales de extracción física y química para palinología (HCl-HF) y al residuo obtenido se lo filtró mediante mallas de $(+10 y+25$ micrones). Las preparaciones definitivas fueron montadas en gelatina glicerina. Los ejemplares fueron estudiados con el microscopio Leitz Wetzlar Germany y Olimpus BX51 (Japan), de la Sección de Paleopalinología y fueron fotografiados con el microscopio óptico de transparencia y el electrónico de barrido (MEB), del Museo Argentino de Ciencias Naturales "Bernardino
Rivadavia”. Para la documentación fotográfica de los ejemplares se utilizó una cámara digital Nikon E4500. Se consideró el sistema de clasificación semi-natural (Pocock, 1962) con la utilización de morfotaxones a nivel de género y especie.

El material estudiado se encuentra depositado en la Colección Palinológica del Museo Regional Provincial "Padre Manuel Jesús Molina" de la ciudad de Río Gallegos, Provincia de Santa Cruz, Argentina (MPM-MP) y los números de catálogo 1943 al 1978. 


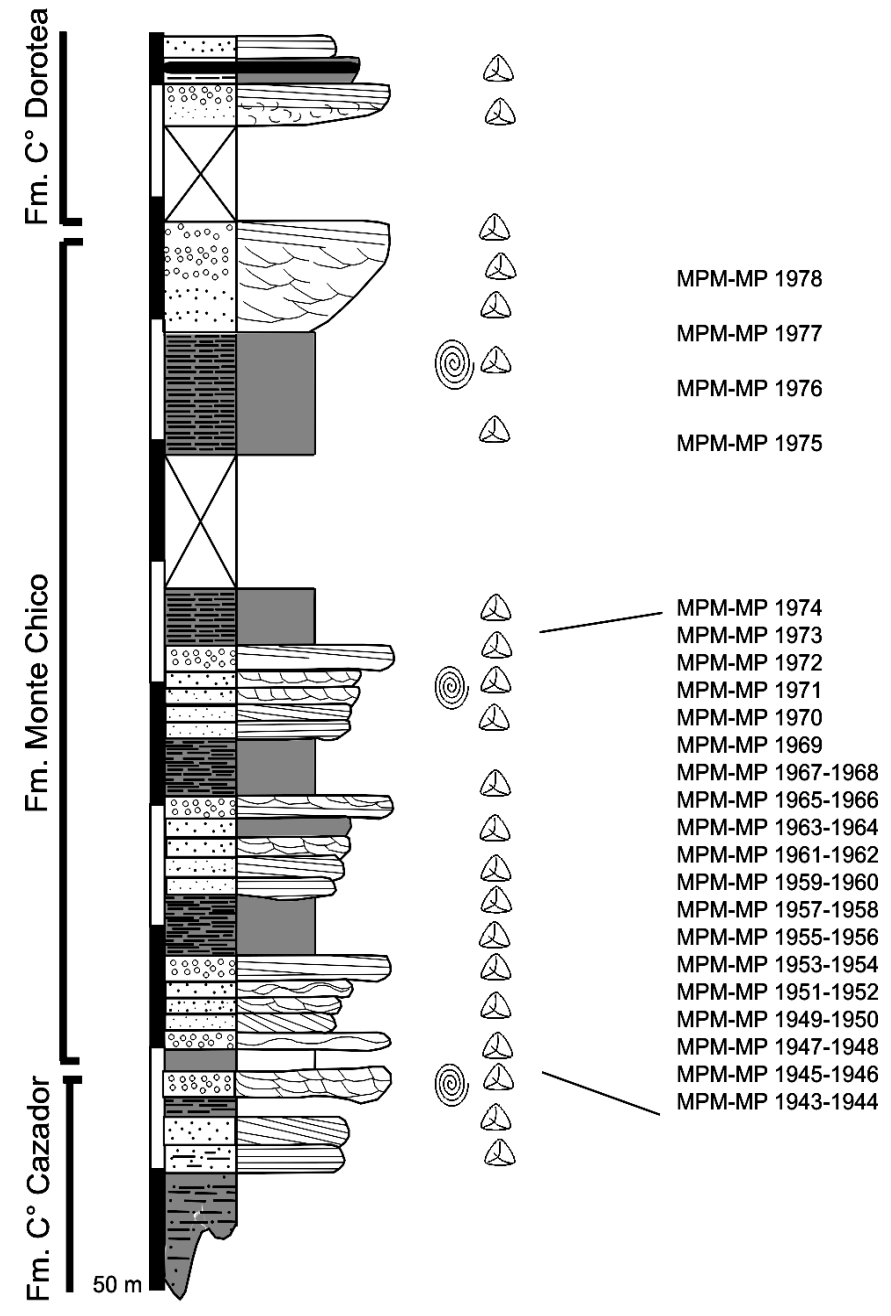

\author{
Litología \\ Cubierto \\ Conglomerado \\ Arenisca gruesa \\ Arenisca fina \\ Fangolitas \\ Pelita \\ Manto de Carbón
}

\section{Estructuras}

$\otimes$ Polen

(e) Trazas fósiles

$\square$ Masiva

근 Coquina

Estratificación entrecruzada

Estratificación laminar planar

Est. entrecruzada de bajo ángulo

$\approx$ Laminación ondulítica

Figura 2. Perfil estratigráfico de la Formación Monte Chico.

Figure 2. Stratigraphic section of the Monte Chico Formation.

\section{PALEONTOLOGÍA SISTEMÁTICA DE TAXA SELECCIONADAS}

Género Araucariacites Cookson, 1947

Especie tipo. Araucariacites australis (Cookson, 1947) Couper, 1953.

\section{Araucariacites australis Cookson, 1947}

(Figura 4A)

Material estudiado. MPM-MP 1947 92,8/20,3; 34,5/103,5; MPM-MP 1946 105,6/7,3; MPM-MP 1958 102,3/4; MPM-MP 1951 110/3,6; 89/4,3; MPM-MP 1957 106/15,4.

Descripción. Grano de polen mediano a grande, de contorno circular a ligeramente oval, no aperturado, generalmente se observan pliegues en la exina. Exina delgada, de 1-1,2 $\mu \mathrm{m}$ de espesor, microgranulada hasta escabrada.

Dimensiones. (3 especímenes medidos) 50 (58) $66 \mu \mathrm{m}$.

Observaciones. Los ejemplares estudiados responden muy bien a la diagnosis de la especie. El rango de variación del tamaño es prácticamente el mismo, aunque el límite superior es considerablemente menor.

Distribución estratigráfica. Presenta una amplia distribución estratigráfica desde el Jurásico temprano al Mioceno de Australia, Nueva Zelanda y Sudamérica.

Afinidad botánica. Familia Araucariaceae.

\section{Género Classopollis Pflug, 1953}

Especie tipo. Classopollis classoides Pflug, 1953.

Classopollis sp. 1

(Figuras 4B-D)

Material estudiado. MPM-MP 1946 100/6; MPM-MP 1955 97/20,4; MPM-MP 1957 105,7/8,7; 103/9,2.

Descripción. Grano de polen rimulado de contorno circular a ovoide en vista ecuatorial y bordes bien redondeados. Rímula de $1,5 \mu \mathrm{m}$. Poro distal de $10 \mu \mathrm{m}$ de diámetro. Engrosamiento 


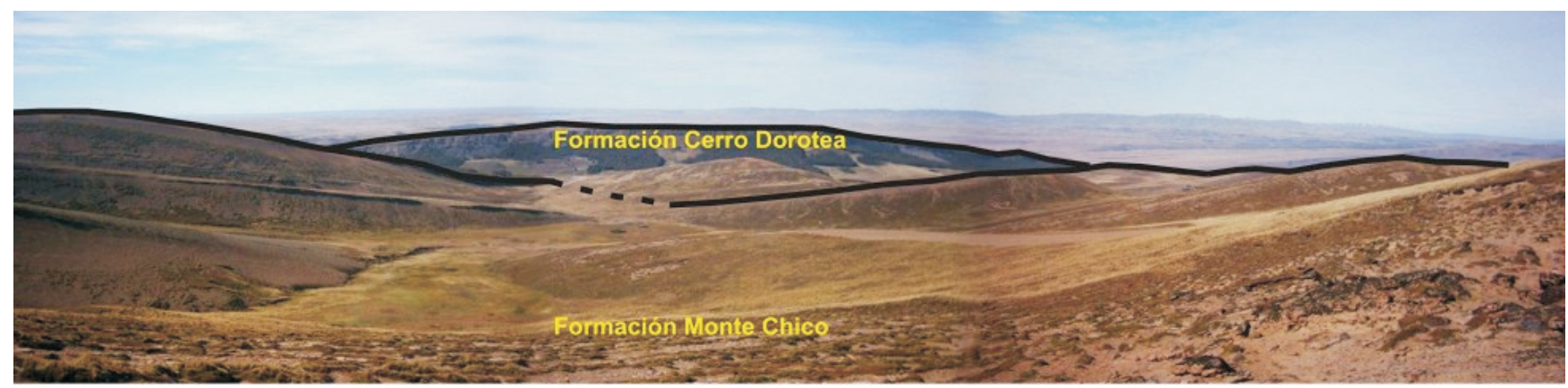

Figura 3. Afloramientos de la Formación Monte Chico en Estancia Laguna Salada. Sem escala.

Figure 3. Outcrops of the Monte Chico Formation in Laguna Salada Farm. Not in scale.

ecuatorial de 3-5 $\mu \mathrm{m}$ de espesor. Exina de 1-1,5 $\mu \mathrm{m}$ de espesor formada por dos capas. Capa externa lisa a microgranulada.

Dimensiones. (6 especímenes medidos) Diámetro ecuatorial 23 $(25,5) 28 \mu \mathrm{m}$; espesor anillo ecuatorial 3-5 $\mu \mathrm{m}$.

Observaciones. Debido a una deficiente preservación no se ha podido determinar el número de endoestrías de la exina. Sin embargo, en algunos ejemplares, las endoestrías (5-6) se observaron apenas marcadas. Estos ejemplares son comparables con los descriptos para el Paleoceno de Formación Salamanca (Archangelsky, 1973) y Maastrichtiano-Paleoceno de las Formaciones Pedro Luro, Jaguel y Lefipán (Ruiz \& Quattrocchio, 1997; Quattrocchio et al., 2005; Barreda com. pers.).

Afinidad botánica. Familia Cheirolepidiaceae.

\section{Género Cycadopites (Wodehouse) Wilson \& Webster, 1946 emend. Herbst, 1965}

Especie tipo. Cycadopites follicularis Wilson \& Webster, 1946.

\section{Cycadopites sp. 1 \\ (Figura 4E)}

Material estudiado. MPM-MP 1953 118/8; MPM-MP 1948 108,5/6; 92,3/8; 105,5/8,2; MPM-MP 1952 102/17,3; MPM-MP $1957106 / 15,4$.

Descripción. Grano de polen monocolpado, prolado, de contorno ecuatorial elíptico, extremos subredondeados a subagudos. Exina lisa, semiescabrada a escabrada y translúcida. Colpo ocupando todo el largo del grano, con los bordes típicamente replegados. Algunos ejemplares se encuentran plegados y parecen más angostos.

Dimensiones. (5 especímenes medidos) Diámetro ecuatorial: 17-29 $\mu \mathrm{m}$; diámetro polar: $34-80 \mu \mathrm{m}$.

Observaciones. Se reúne a una serie de granos monocolpados con un rango de dimensiones muy amplio. Básicamente esta especie se diferencia de Cycadopites nitidus (Balme, 1957) de Jersey, por presentar una exina semiescabrada a escabrada en algunos ejemplares.

Afinidad botánica. Probable afinidad con las Cycadales, Bennettitales y/o Ginkgoales.

\section{Género Dacrycarpites Cookson \& Pike, 1953}

Especie tipo. Dacrycarpites australiensis Cookson \& Pike, 1953.
Dacrycarpites australiensis Cookson \& Pike, 1953

(Figura 4F)

Material estudiado. MPM-MP 1946 97,3/5; MPM-MP 1951 $112,6 / 16,8$.

Descripción. Grano de polen bisacado de cuerpo redondeado a subtriangular con borde crenado en vista polar y elíptica en vista lateral. Exina de 2,5 $\mu \mathrm{m}$ de espesor, con esculturación reticulada de muros anchos a rugulada. Sacos grandes, altos y alargados tangencialmente, $y$ en algunos casos se ponen en contacto entre si. La escultura de los sacos es muy densa, de muros delgados ( $1 \mu \mathrm{m}$ de ancho) con lúmenes de $2 \mu \mathrm{m}$ de diámetro.

Dimensiones. (2 especímenes medidos) Largo total 40-47 $\mu \mathrm{m}$; largo del cuerpo 29-31 $\mu \mathrm{m}$; ancho del cuerpo 29-33 $\mu \mathrm{m}$; largo de los sacos 8-14 $\mu \mathrm{m}$; ancho de los sacos 20-23 $\mu \mathrm{m}$.

Observaciones. Cabe señalar que Cookson \& Pike (1953) hallaron en esta especie tamaños muy variados en formaciones de distinta edad. El material ilustrado por Archangelsky \& Romero (1974) para el Cretácico tardío y Paleoceno de Patagonia es idéntico a nuestros especímenes hallados. Las especies pertenecientes al género Dacrycarpus que son las actuales más similares a la especie fósil, habitan en Australia y Asia.

Distribución estratigráfica. Documentados desde el Cretácico tardío y ampliamente distribuído en el Cenozoico de Sudamérica austral, Australia, Antártida y Nueva Zelanda.

Afinidad botánica. Familia Podocarpaceae, Dacrycarpus (Specht et al., 1992).

\section{Género Gamerroites Archangelsky, 1988}

Especie tipo. Gamerroites volkheimeri Archangelsky, 1988.

Gamerroites psilasaccus (Archangelsky \& Romero, 1974) Archangelsky, 1988

Material estudiado. MPM-MP 1948 93,6/5,3; MPM-MP 1947 90,6/2,1;96,4/4,3; 108,5/21,7.

Descripción. Grano de polen bisacado de cuerpo redondeado en vista polar y elíptico en vista lateral. Exina del cuerpo gruesa de $2 \mu \mathrm{m}$ de espesor, psilada. Sacos grandes y característicamente psilados, implantados distalmente sobre el cuerpo. En algunos especímenes los sacos pueden presentar pequeños pliegues en la base.

Dimensiones. (4 especímenes medidos) Largo total 30-48 $\mu \mathrm{m}$; largo del cuerpo 19-25 $\mu \mathrm{m}$; ancho del cuerpo 20-27 $\mu \mathrm{m}$; largo de los sacos 10-12 $\mu \mathrm{m}$; ancho de los sacos 14-20 $\mu \mathrm{m}$. 
Observaciones. El género Gamerroites y la especie $G$. psilasaccus se caracterizan por presentar los sacos psilados. Los especímenes aquí descriptos presentan similitudes con el material original descripto por Archangelsky \& Romero (1974) para la Formación Bororó, Provincia del Chubut, aunque el rango de tamaño de los granos a veces difiere. Se observaron formas algo deterioradas debido a una posible degradación.

Distribución estratigráfica. Esta especie está ampliamente en Argentina.

Afinidad botánica. Familia Podocarpaceae.

Gamerroites sp. 1

(Figura 4G)

Material estudiado. MPM-MP 1948 115,1/20,4; MPM-MP 1946 90,6/15,2.

Descripción. Grano de polen bisacado, de tamaño mediano. Cuerpo esferoidal, de contorno circular. Exina del cuerpo psilada a escabrada. Los sacos están distalmente insertados, son péndulos, con los bordes de las raíces levemente cóncavos. El largo de los sacos es igual o menor al del cuerpo y su ancho es algo mayor (carácter diploxilonoide). En vista polar, las bases de los sacos no se tocan en el contorno ecuatorial. Sacos ornamentados por muros muy delicados y angostos que en algunos sectores se fusionan y forman un retículo indefinido.

Dimensiones. (1 especímen medido) Largo total $36 \mu \mathrm{m}$; largo del cuerpo $28 \mu \mathrm{m}$; ancho del cuerpo $26 \mu \mathrm{m}$; largo de los sacos $15 \mu \mathrm{m}$; ancho de los sacos $20 \mu \mathrm{m}$.

Observaciones. Sólo se recuperaron dos granos en los niveles basales de la Formación Monte Chico. Gamerroites sp. 1 se aparta de G. psilasaccus, debido a que la forma del cuerpo central del grano es bien redondeada tanto en vista polar como en vista lateral; y los sacos a pesar de ser característicamente psilados, presentan un área de inserción más pequeña sobre el cuerpo, y la exina es más fina y delicada.

Afinidad botánica. Familia Podocarpaceae.

Género Lygistepollenites (Cookson \& Pike) Harris, 1965

Especie tipo. Lygistepollenites florinii (Cookson \& Pike, 1953) Harris, 1965.

\section{Lygistepollenites florinii (Cookson \& Pike, 1953) Stover \& Evans, 1973 \\ (Figuras 4H-I)}

Material estudiado. MPM-MP 1978 101,4/12; MPM-MP 1977 91/7,1; 116/11,6; MPM-MP 1949 98/4,3; MPM-MP 1948 96,3/10,3; MPM-MP 1957 90/11; MPM-MP 1947 91,6/4,3; 96,2/5,8.

Descripción. Grano de polen bisacado de contorno subcircular a oval en vista polar. Cara proximal corrugada a densamente rugulosa, determinando un retículo muy grueso con muros anchos $(2 \mu \mathrm{m})$ y lúmenes muy chicos de tamaño e irregular, de 0,8-1 $\mu \mathrm{m}$ de ancho. La escultura se extiende sobre los sacos en forma contínua. Exina formada por dos capas claramente diferenciadas, de 2-5 $\mu \mathrm{m}$ de espesor, la externa más gruesa que la interna. Sacos pequeños, proyectados distalmente y alargados en forma transversal. Retículo de los sacos laxo, con muros dispuestos en forma radial, lúmenes alargados y anchos, determinando un intrarretículo poligonal a subpoligonal.

Dimensiones. (2 especímenes medidos) Largo total 40-47 $\mu \mathrm{m}$; largo del cuerpo 29-31 $\mu \mathrm{m}$; ancho del cuerpo 29-33 $\mu \mathrm{m}$; largo de los sacos 8-14 $\mu \mathrm{m}$; ancho de los sacos 20-23 $\mu \mathrm{m}$.

Observaciones. Los ejemplares de Patagonia responden a la diagnosis de Lygistepollenites florinii definido para el Terciario de Australia (Cookson \& Pike, 1953). Se encontró gran parecido con los ejemplares descriptos para el Cretácico tardío y Paleoceno de la Formación Bororó, Provincia del Chubut (Archangelsky \& Romero, 1974) y Eoceno de la Formación Río Turbio (Provincia de Santa Cruz) (Romero, 1977). Las medidas del material aquí estudiado son menores que las reportadas por Freile (1972) para el Daniano-Paleoceno de la Formación Cerro Dorotea (Provincia de Santa Cruz). Se trata de una especie presente en bajas proporciones en relación con el resto de las gimnospermas. Distribución estratigráfica. Esta especie se registra ampliamente en Argentina, Chile, Antártida, Australia y Nueva Zelanda, desde el Cretácico tardío al Terciario.

Afinidad botánica. Podocarpaceae, Dacrydium (Specht et al., 1992).

\section{Lygistepollenites sp. 1}

(Figura 4J)

Material estudiado. MPM-MP 1951 105,5/21,4; MPM-MP 1978 101/12; MPM-MP 1947 96,2/5,8; 91,6/4,3; MPM-MP 1946 95,6/6,5.

Descripción. Grano de polen bisacado de contorno subcircular en vista polar. Cara proximal corrugada a densamente rugulosa. La esculturación del cuerpo se extiende sobre los sacos en forma contínua, en algunas ocasiones parece difuso. Exina formada por dos capas claramente diferenciadas, de 2-2,5 $\mu \mathrm{m}$ de espesor, la externa más gruesa que la interna. Sacos pequeños, con el borde redondeado o truncado y proyectados distalmente sobre todo el cuerpo del grano. Retículo de los sacos poco visible debido al pequeño tamaño de éstos.

Dimensiones. (3 especímenes medidos) Largo total 40-45 $\mu \mathrm{m}$; largo del cuerpo 35-38 $\mu \mathrm{m}$; ancho del cuerpo 30-35 $\mu \mathrm{m}$; largo de los sacos 6-7 $\mu \mathrm{m}$; ancho de los sacos 8-10 $\mu \mathrm{m}$.

Observaciones. Los ejemplares descriptos asignados a esta especie se diferencian de Lygistepollenites florinii, por presentar los sacos de menor tamaño y de forma más redondeada a truncada. Afinidad botánica. Familia Podocarpaceae.

\section{Género Microcachyridites Cookson ex Couper, 1953}

Especie tipo. Microcachyridites antarcticus Cookson, 1947. Microcachyridites antarcticus Cookson, 1947 (Figuras 4K-M)

Material estudiado. MPM-MP 1951 86/5,5; MPM-MP 1952 104/3,2; 86/5,2; MPM-MP 1947 107/6,4; MPM-MP 1978 116,5/5,5. Descripción. Grano de polen trisacado, pequeño, con cuerpo circular en vista polar y elíptico en vista lateral. Exina de 1,5-2 $\mu \mathrm{m}$ de espesor. Pared proximal delgada finamente reticulada. Área germinal redondeada o subtriangular. Sacos proyectados distalmente, de pequeño tamaño y retículo denso, con muros finos menores a $0,5 \mu \mathrm{m}$ de ancho. Lúmenes regulares poligonales de hasta $2 \mu \mathrm{m}$ de diámetro.

Dimensiones. (3 especímenes medidos) Largo total $41 \mu \mathrm{m}$; alto total 39-35 $\mu \mathrm{m}$; largo del cuerpo $30 \mu \mathrm{m}$; ancho del cuerpo 29 $\mu \mathrm{m}$; ancho de los sacos 10-15 $\mu \mathrm{m}$; largo de los sacos 15-18 $\mu \mathrm{m}$; espesor de la exina $1,5 \mu \mathrm{m}$. 
Observaciones. Estos granos se asemejan mucho a la descripción original dada por Cookson (1947) en forma, tamaño y dimensiones del retículo. También presentan gran parecido con el material descripto para el Eoceno de la Formación Río Turbio (Romero, 1977) y para el Cretácico tardío y Paleoceno de la Formación Bororó, Provincia del Chubut (Archangelsky \& Romero, 1974).

Distribución estratigráfica. Se encuentra ampliamente distribuída en Argentina, Chile, Antártida, Australia, Nueva Zelanda y Océano Índico, desde el Jurásico al Terciario.

Afinidad botánica. Familia Podocarpaceae. La especie actual más similar a Microcachyridites antarcticus es Microcachrys terragona presente en Tasmania.

\section{Género Phyllocladidites Cookson, 1947}

Especie tipo. Phyllocladidites mawsonii Cookson, 1947 ex Couper, 1953.

\section{Phyllocladidites mawsonii Cookson, 1947 ex Couper, 1953} (Figuras 4N-P)

Material estudiado. MPM-MP 1946 111/23,2; 110/17,3; MPM-MP 1949 104,6/5,7; MPM-MP 1945 112/6; MPM-MP 1947 0,7/146,4. Descripción. Grano de polen bisacado isodiamétrico, de pequeño tamaño, cuerpo de forma subcircular a esferoidal en vista polar, con dos tubérculos proximales. Sacos pequeños ubicados distalmente, más anchos que altos, bordeando la zona germinal. A veces suelen tener una leve constricción en la base. Pared de los sacos ornamentada con microgránulos pobremente desarrollados. El cuerpo presenta una exina engrosada y microperforada con aspecto macizo.

Dimensiones. (4 especímenes medidos) Largo total $30 \mu \mathrm{m}$; alto total $33 \mu \mathrm{m}$; largo del cuerpo 24 (25) $26 \mu \mathrm{m}$; ancho del cuerpo 22 (24) $26 \mu \mathrm{m}$; alto del cuerpo $24 \mu \mathrm{m}$; ancho del saco 8 (12) 16 $\mu \mathrm{m}$; largo del saco 9 (10) $11 \mu \mathrm{m}$; alto del saco $10 \mu \mathrm{m}$; espesor de la exina 1,6-2 $\mu \mathrm{m}$.

Observaciones. Este material coincide perfectamente con el material tipo definido para el Terciario de las Islas Kerguelen (Cookson, 1947). También se encontraron semejanzas con Phyllocladidites mawsonii de la Formación Río Turbio (Eoceno), (Romero, 1977); sin embargo, el límite superior de las dimensiones es algo más amplio. Los sacos por lo general, aparecen plegados sobre el cuerpo. En este trabajo se siguió el criterio de Playford \& Dettmann (1978), y todo el material aquí estudiado se ubica dentro de la especie P. mawsonii, ya que estos autores concluyen que no existen diferencias morfológicas o estratigráficas para separarla de $P$. ruei.

Distribución estratigráfica. La especie se encuentra distribuida en Argentina, Antártida, Chile, Australia, Nueva Zelanda y Estados Unidos, desde el Maastrichtiano al Cenozoico. $\mathrm{Su}$ registro llega a la actualidad, aunque posteriormente al Mioceno se restringía a la región occidental de Tasmania, donde actualmente se desarrolla (Playford \& Dettmann, 1978).

Afinidad botánica. Familia Podocarpaceae. Similar a Dacrydium frankliinii conforme Couper (1953) y Cookson \& Cranwell (1967), Lagarostrobus (Specht et al., 1992).

Género Podocarpidites Cookson, 1947

Especie tipo. Podocarpidites ellipticus Cookson, 1947 (Couper, 1953).

\section{Podocarpidites elegans Romero, 1977}

(Figura 4Q)

Material estudiado. MPM-MP 1947 0,5/145,6; 97/3,7; 88/5,4; 87/3,2; 97,3/1,8; MPM-MP 1957 101,8/13.

Descripción. Grano de polen bisacado de cuerpo pequeño, ovalado en vistas polar y lateral. Pared proximal delgada, formada por un retículo muy fino, con muros delicados muy anastomosados y lúmenes estrechos e irregulares, menores a $1 \mu \mathrm{m}$ de diámetro. Sacos pequeños, algo aplanados y proyectados distalmente limitando un área germinal estrecha. Generalmente el ancho de los sacos es menor que el del cuerpo. Intrarretículo de los sacos con muros gruesos, de 1 $\mu \mathrm{m}$ de ancho, poco o muy denso, presentando lúmenes chicos o medianos.

Dimensiones. (3 especímenes medidos) Largo total 30 (35) $40 \mu \mathrm{m}$; Largo del cuerpo 12 (17) $22 \mu \mathrm{m}$; Ancho del cuerpo 20 (24) $28 \mu \mathrm{m}$; Alto del cuerpo 10 (15) $20 \mu \mathrm{m}$; Largo de los sacos 11 (14) $17 \mu \mathrm{m}$; Ancho de los sacos 16 (19) $22 \mu \mathrm{m}$.

Observaciones. Coincide con los caracteres diagnósticos dados para el material tipo de la Formación Río Turbio (Eoceno), Provincia de Santa Cruz (Romero, 1977); también con los ejemplares asignados al Maastrichtiano de Patagonia (Archangelsky \& Romero, 1974). Son granos relativamente escasos con respecto al resto de las podocarpáceas y se presentan en forma aislada.

Distribución estratigráfica. Esta especie ha sido citada para Argentina y Antártida desde el Cretácico tardío al Mioceno. Afinidad botánica. Familia Podocarpaceae.

\section{Podocarpidites ellipticus Cookson, 1947} (Figura 4R)

Material estudiado. MPM-MP 1949 96,1/18,2; MPM-MP 1946 97/10; MPM-MP 1962 108/7,6.

Descripción. Grano de polen bisacado, de forma elíptica en vista polar. Exina proximal del cuerpo granular punteada con cresta marginal desarrollada. Sacos delicados, frecuentemente arrugados y unidos lateralmente, sobresaliendo del contorno ecuatorial del cuerpo central (carácter diploxilonoide). Generalmente el ancho del cuerpo sobrepasa el alto. Intrarretículo de los sacos irregular, poco definido y de malla pequeña.

Dimensiones. (4 especímenes medidos), largo total 36-54 $\mu \mathrm{m}$; alto total $27 \mu \mathrm{m}$; largo del cuerpo $25 \mu \mathrm{m}$; ancho del cuerpo $26 \mu \mathrm{m}$; ancho del saco $15 \mu \mathrm{m}$; largo del saco $28 \mu \mathrm{m}$; espesor de la exina $2 \mu \mathrm{m}$.

Observaciones. Los especímenes aquí estudiados concuerdan con las características morfológicas y rango de dimensiones dadas para el material del Jurásico medio de Picún Leufú, Provincia de Neuquén (Menéndez, 1968); aunque en la Formación Monte Chico algunos de ellos se encuentran en mal estado de conservación. Podocarpidites cf. P. ellipticus citada para el Mesozoico tardío del SE de Australia (Dettmann, 1963) presenta un rango de dimensiones algo mayores.

Distribución estratigráfica. Especie distribuida mundialmente y citada en Argentina, Antártida, Australia, Nueva Zelanda y Estados Unidos.

Afinidad botánica. Familia Podocarpaceae, Podocarpus y algunas especies de Dacrydium conforme Dettmann (1989). 


\section{Podocarpidites marwickii Couper, 1953}

(Figura 4S)

Material estudiado. MPM-MP 1947 86/19,4; MPM-MP 1955 97/21; 93,5/4; MPM-MP 1956 53,7/99,3.

Descripción. Grano de polen bisacado isodiamétrico, elíptico en vista polar. Pared proximal delgada con un retículo muy fino y denso, con muros muy delgados (menores de $1 \mu \mathrm{m}$ ) que limitan espacios poligonales, comúnmente alargados, más delgados que los muros. Zona germinal transversalmente alargada y ancha. Sacos semicirculares en vista polar, con un retículo abierto y fino, a veces con muros ciegos especialmente en el extremo del saco. Muros delgados $(<$ de $1 \mu \mathrm{m})$ y dejan espacios poligonales de hasta $3 \mu \mathrm{m}$ de diámetro.

Dimensiones. (4 especímenes medidos) Largo total 56 (66) 76 $\mu \mathrm{m}$; largo del cuerpo 30 (39) $52 \mu \mathrm{m}$; ancho del cuerpo 32 (40) $48 \mu \mathrm{m}$; alto del cuerpo 15 (22) $27 \mu \mathrm{m}$; largo de los sacos 20 (28) $36 \mu \mathrm{m}$; ancho de los sacos 25 (30) $34 \mu \mathrm{m}$.

Observaciones. Este material responde a la diagnosis específica de Couper (1953), coincidiendo en forma, escultura del cuerpo y de los sacos, intrarretículo de los sacos y tamaño. El ancho de los sacos casi siempre es igual al del cuerpo, en muy pocos casos son más anchos que el cuerpo. Éstos ejemplares se compararon con Podocarpidites marwickii del Maastrichtiano de Patagonia (Freile, 1972), Paleoceno de la Provincia de Santa Cruz (Archangelsky \& Romero, 1974), Eoceno de Chile (Troncoso \& Barrera, 1980) y Terciario de Collón Curá (Baldoni, 1987) y se encontraron dimensiones muy semejantes. En cambio, los granos de Río Turbio (Romero, 1977) son algo más grandes.

Distribución estratigráfica. Esta especie se registra en Argentina, Chile, Antártida, Nueva Zelanda, Australia y Estados Unidos, desde el Maastrichtiano al Terciario.

Afinidad botánica. Familia Podocarpaceae.

Podocarpidites microreticuloidata Cookson, 1947

(Figuras 4T; 5A)

Material estudiado. MPM-MP 1949 111,5/16,4; MPM-MP $195088 / 20,4$.

Descripción. Grano de polen bisacado isodiamétrico, de cuerpo circular en vista polar. Sacos implantados en la mitad distal del grano, a ambos lados de la zona germinal. Calota relativamente delgada, formada por un retículo muy fino, de muros delgados, $0,7-0,8 \mu \mathrm{m}$ de ancho, anastomosados, dejando entre sí espacios alargados y estrechos. La zona germinal es alargada transversalmente, presentando los bordes bien marcados. Sacos relativamente pequeños y de retículo muy denso; por lo general, su ancho es menor que el del cuerpo. Retículo de los sacos de muros delgados y muy anastomosados, con lúmenes muy pequeños y subpoligonales, de hasta $2,5 \mu \mathrm{m}$ de diámetro, dando un aspecto cerebroide.

Dimensiones. (3 especímenes medidos) Largo total 36 (45) 54 $\mu \mathrm{m}$; largo del cuerpo 30 (36) $42 \mu \mathrm{m}$; ancho del cuerpo 32 (38) $44 \mu \mathrm{m}$; alto del cuerpo 19 (22) $25 \mu \mathrm{m}$; largo de los sacos 16 $(18,5) 21 \mu \mathrm{m}$; ancho de los sacos 20 (28) $34 \mu \mathrm{m}$; espesor de la exina $1-1,5 \mu \mathrm{m}$.

Observaciones. El tamaño del cuerpo y el ancho de la zona germinal varían en nuestro material en un rango más reducido que el indicado en la descripción original. Las dimensiones generales de los granos aquí analizados, son menores y el ancho de los sacos ligeramente mayor. Archangelsky \& Romero (1974) y Romero (1977) señalaron como elementos diagnósticos la forma del cuerpo y esculturación de los sacos, con los que se asemejan al material aquí estudiado. Las formas registradas en la Formación Río Turbio (Eoceno) (Romero, 1977) presentan el cuerpo más circular y el retículo de los sacos más pequeño y denso que nuestros especímenes.

Distribución estratigráfica. Los registros conocidos corresponden a Argentina, Antártida, Chile, Australia y Nueva Zelanda.

Afinidad botánica. Familia Podocarpaceae. No existen formas similares actuales (Couper, 1953).

\section{Podocarpidites cf. P. microreticuloidata Cookson, 1947} (Figuras 5B-C)

Material estudiado. MPM-MP 1945 93,2/19,3.

Descripción. Grano de polen bisacado isodiamétrico, de cuerpo circular en vista polar. Sacos implantados en la mitad distal del grano. Carácter diploxilonoide. Sacos medianos a grandes, muy reticulados con aerolas pequeñas y delicadas.

Dimensiones. ( 1 especímen medido) Largo total $82 \mu \mathrm{m}$; largo de los sacos $35 \mu \mathrm{m}$; largo del cuerpo $30 \mu \mathrm{m}$; alto total $60 \mu \mathrm{m}$; alto de los sacos $60 \mu \mathrm{m}$; alto del cuerpo $40 \mu \mathrm{m}$.

Observaciones. Se presenta en los niveles inferiores de la Formación Monte Chico en muy bajas proporciones y se diferencia de Podocarpidites microreticuloidata (Figuras 4LM) por presentar mayores dimensiones generales y porque el infraretículo de los sacos es algo más fino y delicado.

Afinidad botánica. Familia Podocarpaceae.

Género Trisaccites (Cookson) emend. Menéndez, 1968

Especie tipo. Trisaccites microsaccatum (Couper) Couper, 1960.

Trisaccites microsaccatum (Couper) Couper, 1960

(Figuras 5D-E)

Material estudiado. MPM-MP 1957 105/9,2; MPM-MP 1977 $87,7 / 14,1$.

Descripción. Grano de polen de pequeño tamaño, con la pared distal aplanada, subtriangular en vista polar. Pared proximal delgada, reticulada, de 1,2 $\mu \mathrm{m}$ de espesor. Area germinal triangular. Sacos pequeños, de base amplia, implantados sobre la cara distal, poniéndose en contacto las bases, contraídos hacia el interior del cuerpo. Retículo de los sacos muy denso y grueso. Dimensiones. (3 especímenes medidos) Diámetro total 17 (21) 25 $\mu \mathrm{m}$; ancho del saco 7 (10) $13 \mu \mathrm{m}$; profundidad del saco 5,5 (7) $9 \mu \mathrm{m}$. Observaciones. El material analizado por Romero (1977) para el Eoceno de la Formación Río Turbio, Provincia de Santa Cruz, presenta un rango de dimensiones algo mayores que el que se describe en este trabajo; y el aspecto de la pared llega a ser reticulada a granulada, mientras que aquí sólo se observa una pared reticulada. Los escasos especímenes estudiados se encuentran algo distorsionados debido a la mala preservación del material; no obstante, la morfología y dimensiones de nuestros ejemplares se ajustan perfectamente al material descripto por Archangelsky \& Romero (1974) para el Cretácico tardío y Paleoceno de Patagonia.

Distribución estratigráfica. Esta especie ha sido registrada en Argentina, Chile, Antártida, Australia y Nueva Zelanda desde el Jurásico medio al Cenozoico.

Afinidad botánica. Familia Podocarpaceae. 

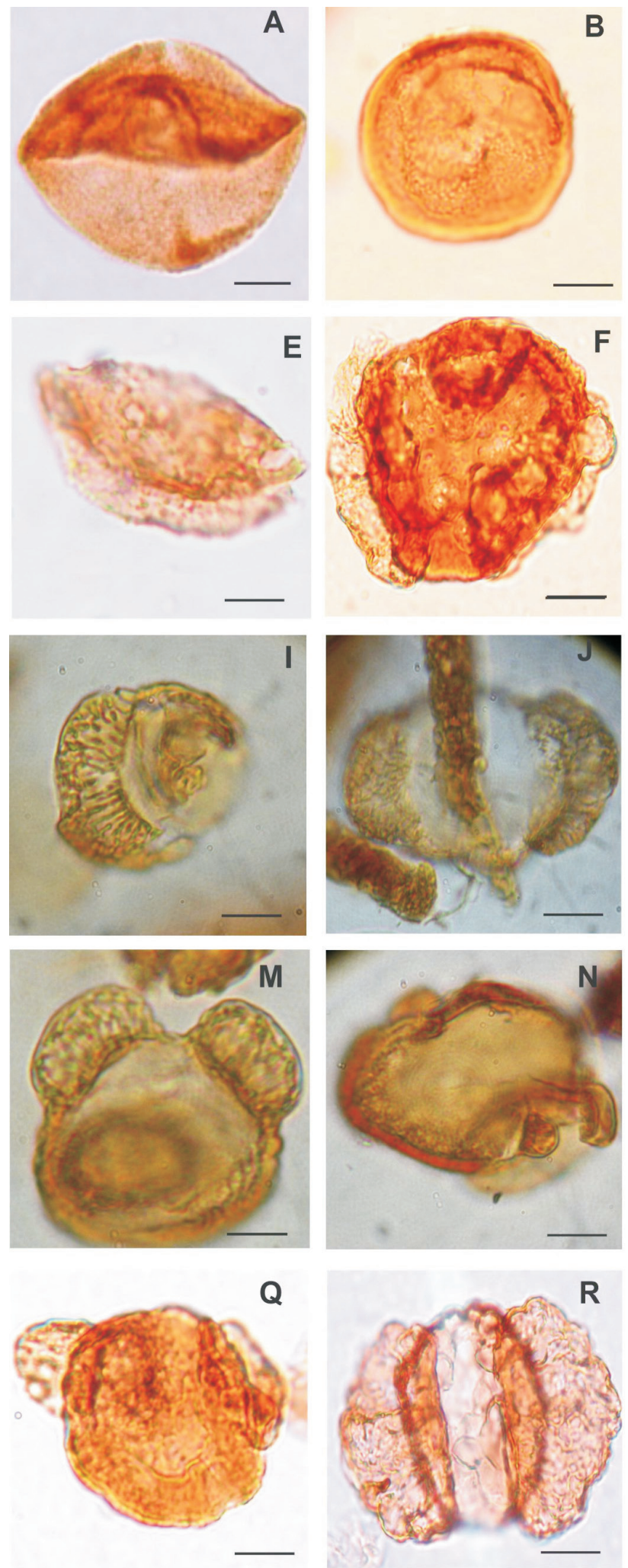

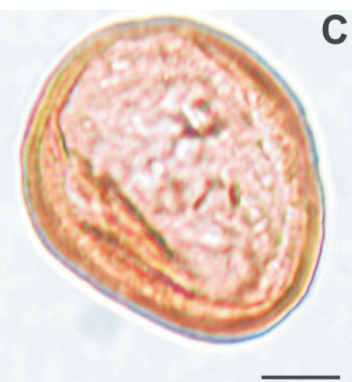

C
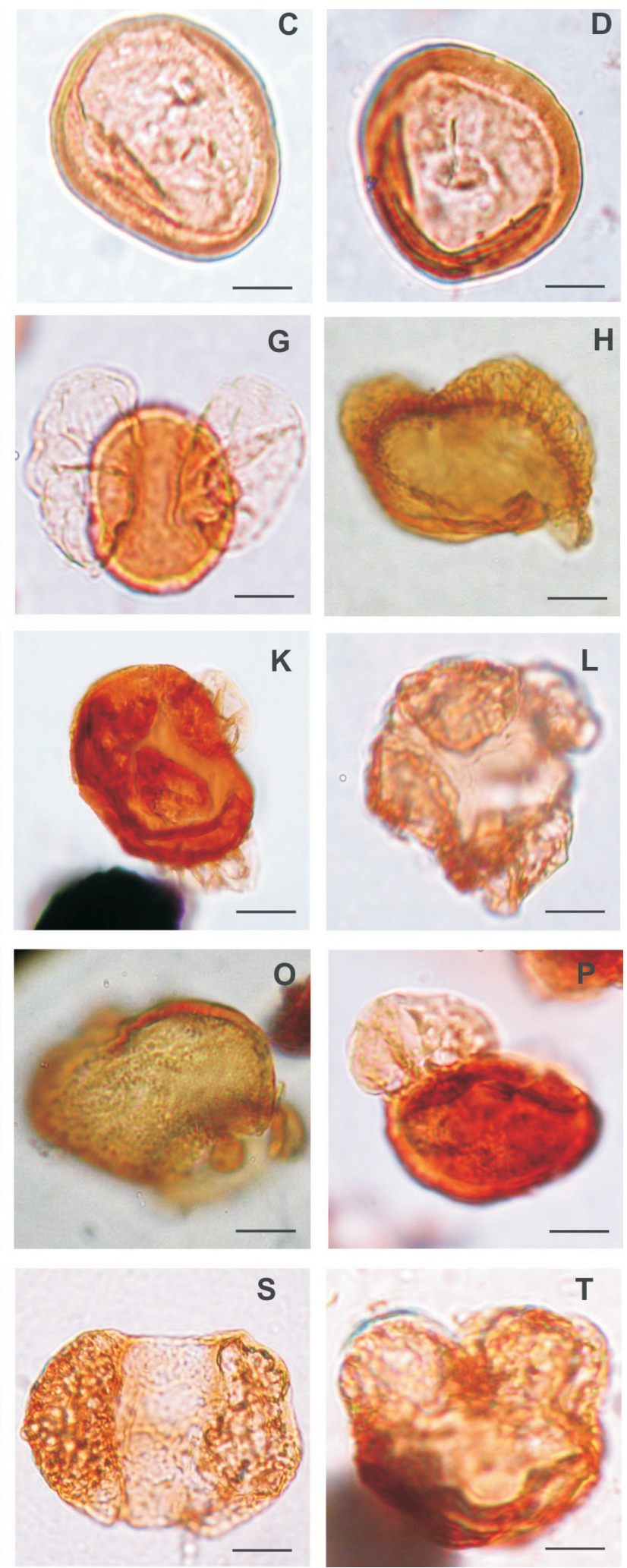

Figura 4. A, Araucariacites australis, MPM-MP 1947 92,5/21. B-D, Classopollis sp. 1, B, MPM-MP 1957 105,7/8,7; C, MPM-MP 1946 100/6. D, MPM-MP 1955 97/20,4. E, Cycadopites sp. 1, MPM-MP 1953 118/8. F, Dacrycarpites australiensis, MPM-MP 1946 97,3/5. G, Gamerroites sp. 1, MPM-MP 1948 115,1/20,4. H-I, Lygistepollenites florinii, MPM-MP 1978 101,4/12. J, Lygistepollenites sp. 1, MPM-MP 1951 105,5/21,4. K-M, Microcachyridites antarcticus, K, MPM-MP 1951 86/5,5; L, MPM-MP 1952 104/3,2; M, MPM-MP 1952 86/5,2. N-P, Phyllocladidites mawsonii, N-O, MPM-MP 1946 111/23,2; P, MPM-MP 1946 110/17,3. Q, Podocarpidites elegans, MPM-MP 1947 0,5/145,6. R, Podocarpidites ellipticus, MPM-MP 1949 96,1/18,2. S, Podocarpidites marwickii, MPM-MP 1947 86/19,4. T, Podocarpidites microreticuloidata, MPM-MP 1949 $111,5 / 16,4$. Escalas $=10 \mu \mathrm{m}$.

Figure 4. Photomicrographs of the study species. Scale bars $=10 \mu \mathrm{m}$. 

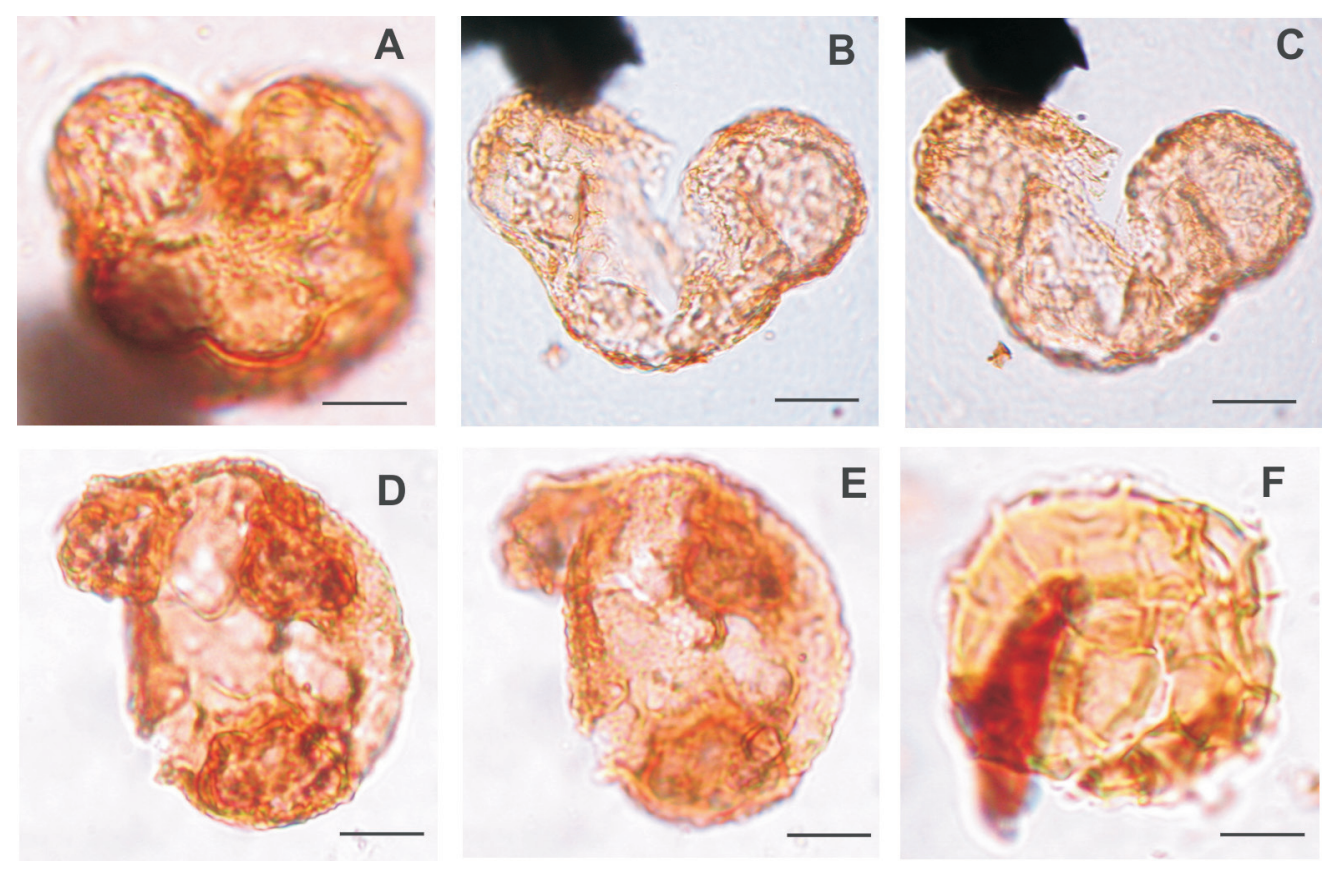

Figura 5. A, Podocarpidites microreticuloidata, MPM-MP 1949 111,5/16,4. B-C, Podocarpidites cf. P. microreticuloidata, MPM-MP 1945 93,2/19,3. D-E, Trisaccites microsaccatum, MPM-MP 1957 105/9,2. F, Acritarco indeterminado. Escalas $=10 \mu \mathrm{m}$.

Figure 5. Photomicrographs of the study species. Scale bars $=10 \mu \mathrm{m}$.

\section{DISCUSIÓN}

En esta publicación se presenta el estudio sistemático de los granos de polen pertenecientes a las gimnospermas de la Formación Monte Chico. Los grupos morfológicos abordados corresponden a granos inaperturados, bisacados y trisacados (15 especies en total). El grupo vegetal mejor representado a lo largo de la columna estratigráfica de la Formación Monte Chico es la familia Podocarpaceae registrándose con las especies referidas al género Podocarpidites, Dacrycarpites, Gamerroites, Lygistepollenites, Microcachyridites, Phyllocladidites y Trisaccites constituyendo el 20-25\% de abundancia relativa en la asociación de granos de polen de gimnospermas. Los granos bisacados, en la asociación son bastante abundantes en casi todos los niveles, encontrándose en un 17-20\% de abundancia relativa, mientras que los granos trisacados son escasos, estando representados en un 4-5\% de abundancia relativa. Estos elementos (bisacados y trisacados) componen los elementos arbóreos (Familias Podocarpaceae, Araucariaceae y Cheirolepidiaceae) de la comunidad vegetal de la formación (Llorens, 2005; Archangelsky \& Villar de Seoane, 2005).

Las familias Araucariaceae, Cheirolepidiaceae y Cycadaceae están representas por los géneros Araucariacites, Classopollis y Cycadopites respectivamente. El primer género aparece sólo en algunos niveles y en forma muy escasa (menos del $2 \%$ de abundancia relativa) y no presenta importancia bioestratigráfica. Las Cheirolepidiáceas son más abundantes que las Araucariáceas y Cycadales y están representadas en la Formación Monte Chico por la especie Classopollis sp. 1. Se registran con frecuencia en los niveles medios y superiores de la sección estudiada con el $15-18 \%$ de abundancia relativa.

La abundancia relativa de las gimnospermas en general, dentro de la microflora, junto a las esporas de pteridófitas y briófitas, que han sido objeto de estudio en una contribución anterior, sugieren un entorno paleoambiental de alta humedad en el medio. Por su parte, uno de los resultados arrojados en el análisis de las esporas es el hallazgo de Sphagnaceae (Stereisporites antiquasporites), que junto a los granos de polen aquí estudiados, indicarían la presencia de suelos inundados o muy húmedos. El desarrollo de cuerpos de agua dulce a salobre en las inmediaciones también estaría soportado, al menos localmente por la presencia de Botryococcus y Pediastrum (ilustrados en Povilauskas, 2011).

Las bajas frecuencias de las familias Podocarpaceae y Araucariaceae respecto a las esporas de pteridófitas y briófitas, observadas a lo largo de toda la unidad estudiada, parecen sugerir que los bosques se habrían desarrollado en altos topográficos a cierta distancia de la cuenca de depositación.

Como resultado, se interpreta que toda la unidad se habría depositado bajo un régimen costero marginal, probablemente somero, bajo condiciones de clima cálido y al menos localmente húmedo.

\section{AGRADECIMIENTOS}

La autora agradece a M. Arai y a L. Antonioli por los comentarios críticos que permitieron mejorar el manuscrito original, a L. Balarino por proveer programas informáticos para realizar este trabajo, a $\mathrm{O}$. Cárdenas por la ayuda brindada en el procesamiento de las muestras palinológicas, al CONICET y a la Agencia Nacional de Promoción Científica y Tecnológica por el apoyo económico brindado (PICT 32320).

\section{REFERENCIAS}

Archangelsky, S. 1973. Palinología del Paleoceno de Chubut 1. Descripciones sistemáticas. Ameghiniana, 10:339-399. 
Archangelsky, S. \& Romero, E.J. 1974. Polen de gimnospermas (coníferas) del Cretácico Superior y Paleoceno de Patagonia. Ameghiniana, 11:217-236.

Archangelsky, S. \& Villar de Seoane, L. 2005. Estudios palinológicos del Grupo Baqueró (Cretácico Inferior), provincia de Santa Cruz, Argentina. IX Polen bisacado de Podocarpaceae. Revista Española de Paleontología, 20:37-56.

Baldoni, A.M. 1987. Estudios palinológicos de la zona de Collón Cura, Provincia del Neuquén, sobre elementos del Terciario Inferior y redepositados del Cretácico Inferior. Revista Española de Micropaleontología, 19:367-411.

Cookson, I.C. 1947. Plant microfossils from the lignites of Kerguelen archipiélago. British, Australian and New Zealand Antarctic Research Expedition 1929-31, Reports A, 2:129-142.

Cookson, I.C. \& Cranwell, L.M. 1967. Lower Tertiary microplankton, spores and pollen grains from southermost Chile. Micropaleontology, 13:204-216.

Cookson, I.C. \& Pike, K.M. 1953. The Tertiary occurrence and distribution of Podocarpus (Section Dacrycarpus) in Australia and Tasmania. Australian Journal of Botany, 1:71-82.

Couper, R.A. 1953. Upper Mesozoic and Cainozoic spores and pollen grains from New Zealand. New Zealand Geological Survey Palynology Bulletin, 22:1-77.

Couper, R.A. 1960. New Zealand Mesozoic and Cainozoic plant microfossils. New Zealand Geological Survey Palaeontological Bulletin, 32:1-87.

Dettmann, M.E. 1963. Upper Mesozoic microfloras from southeastern Australia. Proceedings of the Royal Society of Victoria, 77:1-148.

Dettmann, M.E. 1989. Antarctica: Cretaceous cradle of austral temperate rainforests? Geological Society Special Publication, 47:89-105.

Freile, C. 1972. Estudio palinológico de la Formación Cerro Dorotea (Maastrichtiano-Paleoceno) de la provincia de Santa Cruz. Revista Museo de La Plata, Sección Paleontología, 6:39-63.

Llorens, M. 2005. Palinología de la Formación Punta del Barco, Cretácico Inferior de Santa Cruz. Tesis doctoral, Universidad Nacional de La Plata, 210 p.

Malumián, N. \& Panza, J. 1996. Hoja Geológica Yacimiento Río Turbio 5172 III. Buenos Aires, Servicio Geológico Minero Argentino, (Boletín 247).
Menéndez, C.A. 1968. Estudio palinológico del Jurásico Medio de Picun Leufu, Neuquén. Ameghiniana, 5:379-405.

Pflug, H.D. 1953. Zur Entstehung und Entwicklung des angiospermiden pollens in der Erdgeschichte. Palaeontographica Abteilung B, 95:60-171.

Playford, G. \& Dettmann, M.E. 1978. Pollen of Dacrydium franklinii Hook. and comparable early Tertiary microfossils. Pollen et Spores, 20:513-534.

Pocock, S.A. 1962. Microfloral analysis and age determinations of strata at the Jurassic-Cretaceous boundary in the western Canada Plains. Palaeontographica Abteilung B, 111:1095.

Povilauskas, L. 2011. Palinología de la Formación Monte Chico (Cretácico tardío) de la Provincia de Santa Cruz, Argentina: Esporas. Revista Brasileira de Paleontologia, 14:255-268. doi:10.4072/rbp.2011.3.05

Quattrocchio, M.; Martínez, M.A.; Carpinelli Pavisich, A. \& Volkheimer, W. 2005. Early Cretaceous palynostratigraphy, palynofacies and paleoenvironments of well sections in northeaster Tierra del Fuego, Argentina. Cretaceous Research, 27:584-602. doi:10.1016/j. cretres.2005.11.012

Romero, E.J. 1977. Polen de gimnospermas y fagáceas de la Formación Río Turbio (Eoceno), Santa Cruz, Argentina. Buenos Aires, Fundación para la Educación, la Ciencia y la Cultura, 219 p.

Ruiz, L.C. \& Quattrocchio, M.E. 1997. Estudio palinológico de la Formación Pedro Luro (?Maastrichtiano-Paleoceno) en la Cuenca del Colorado, Republica Argentina. Parte 1: Esporas triletes, Laevigati, Murornati, Tricassati, Cingulati y Zonati. Revista Española de Micropaleontología, 29:13-29.

Specht, M.; Pedarnig, J.D.; Heck1, W.M. \& Hänsch, T.W. 1992. Scanning plasmon near-field microscope. Physical Review Letters, 68:476479. doi: 10.1103/PhysRevLett.68.476

Stover, L. E. \& Evans, R. 1973. Upper Cretaceous-Eocene spore-pollen zonation, offshore Gippsland Basin, Australia. Geological Society of Australia, Special Publication, 4:55-72.

Troncoso, A. \& Barrera, E. 1980. Polen del Eoceno de Osorno. Boletín Museo Nacional de Historia Natural, 37:179-203.

Wilson, L. \& Webster, R. 1946. Plant microfossils from a Fort Union coal of Montana. American Journal of Botany, 33:271-278.

Received in October, 2010; accepted in March, 2012.

Apéndice 1. Lista de especies identificadas.

Appendix 1. List of species identified.

Gimnospermas

Araucariacites australis Cookson, 1947 (Figura 4A)

Classopollis sp. 1 (Figuras 4B-D)

Cycadopites sp. 1 (Figura 4E)

Dacrycarpites australiensis Cookson \& Pike, 1953 (Figura 4F)

Gamerroites psilasaccus (Archangelsky \& Romero, 1974) Archangelsky 1988 (no ilustrado)

Gamerroites sp. 1 (Figura 4G)

Lygistepollenites florinii (Cookson \& Pike, 1953) Stover \& Evans, 1973 (Figuras 4H-I)

Lygistepollenites sp. 1 (Figura 4J)

Microcachyridites antarcticus Cookson, 1947 (Figuras 4K-M)

Phyllocladidites mawsonii Cookson, 1947 ex Couper, 1953 (Figuras 4N-P)

Podocarpidites elegans Romero, 1977 (Figura 4Q)

Podocarpidites ellipticus Cookson, 1947 (Figura 4R)

Podocarpidites marwickii Couper, 1953 (Figura 4S)

Podocarpidites microreticuloidata Cookson, 1947 (Figuras 4T; 5A)

Podocarpidites cf. P. microreticuloidata Cookson, 1947 (Figuras 5B-C)

Trisaccites microsaccatum Couper, 1960 (Figuras 5D-E)

Dinoflagelados

Acritarcos indet. (Figura 5F) 